\title{
Inhibition of cardiotrophin-1 overexpression is involved in the anti-fibrotic effect of Astrogaloside IV
}

\author{
GUIZHI JIA ${ }^{1}$, BIN LENG ${ }^{2}$, HONGXIN WANG ${ }^{2}$ and HONGLIANG DAI ${ }^{3}$ \\ Departments of ${ }^{1}$ Physiology and ${ }^{2}$ Pharmacology, Jinzhou Medical University; ${ }^{3}$ Department of Community Health Nursing, \\ School of Nursing, Jinzhou Medical University, Jinzhou, Liaoning 121001, P.R. China
}

Received February 20, 2017; Accepted September 19, 2017

DOI: $10.3892 / \mathrm{mmr} .2017 .7676$

\begin{abstract}
Astragaloside IV (AsIV), one of the major active ingredients in Astragalus membranaceus, has demonstrated remarkable antifibrotic effects via its antioxidative activity. Cardiac fibrosis is an important pathological mechanism during cardiac remodelling associated with heart failure. In the present study, the mechanism underlying the antifibrotic effect of AsIV upon isoprenaline (ISO) stimulation was investigated. AsIV significantly improved cardiac fibrosis in vivo and dose-dependently inhibited ISO-induced CF proliferation in vitro. The ISO-triggered elevation in reactive oxygen species (ROS) levels was remarkably inhibited by AsIV, as well as ROS scavenger $N$-acetylcysteine (NAC), and not affected by cardiotrophin-1 (CT-1) knockdown. In addition, AsIV effectively reversed ISO-induced upregulation of CT-1 expression, which was blunted by pretreatment with NAC. Cardiac fibroblast $(\mathrm{CF})$ proliferation and collagen I overexpression induced by ISO stimulation were effectively abrogated by AsIV, NAC, and CT-1 small interfering RNA transfection. Taken together, these results demonstrated that AsIV was able to effectively inhibit ISO-induced CF proliferation and collagen production through negative regulation of ROS-mediated CT-1 upregulation.
\end{abstract}

\section{Introduction}

Cardiac fibrosis can be caused by a variety of cardiovascular disorders, such as hypertension, ischemic injury, valvular heart disease (1-3). Cardiac fibrosis, which is characterized by enhanced cardiac fibroblast (CF) proliferation and excess

Correspondence to: Professor Hongxin Wang, Department of Pharmacology, Jinzhou Medical University, No. 40, Section 3, Songpo Road, Jinzhou, Liaoning 121001, P.R. China

E-mail: jyhxwang@163.com

Dr Hongliang Dai, Department of Community Health Nursing, School of Nursing, Jinzhou Medical University, No. 40, Section 3, Songpo Road, Jinzhou, Liaoning 121001, P.R. China

E-mail: jy2006hldai@sohu.com

Key words: astragaloside IV, isoprenaline, cardiac fibrosis, reactive oxygen species, cardiotrophin-1 production of extracellular matrix (ECM) such as collagen, plays a pivotal role in pathological cardiac remodelling and is an important determinant of many fatal cardiovascular events, such as heart failure, severe arrhythmias and sudden cardiac death (4-6). Pathologically, cardiac fibrosis is characterized by excessive collagen accumulation and fibroblast deposition in the heart, thereby leading to reduction of cardiac muscle compliance, filling impairment, and ultimately congestive heart failure $(4,7)$. Therefore, preventing or slowing the progression of cardiac fibrosis is beneficial for the prognosis of cardiovascular disorders.

Cardiotrophin-1 (CT-1) is a member of the interleukin 6 superfamily. Although initially, CT-1 was regarded as an adaptive response factor mediating cell surviving diverse adverse stimiuli, accumulating in vitro evidences suggest that CT-1 also acts as a profibrotic cytokine in cardiac fibroblasts $(8,9)$. Meanwhile, existing in vivo experimental and clinical data also show that CT-1 is an important pro-fibrotic molecule in the heart (10). Regarding the molecular regulation of CT-1 expression, a previous study has shown that reactive oxygen species (ROS) acts as a pivotal mediator for the upregulation of CT-1 (11).

As an important 'Qi-invigorating' medical herb, A. membranaceus is widely used in traditional Chinese medicine for the treatment of cardiovascular diseases, hepatitis, kidney disease, and skin diseases (12-14). Astragaloside IV (AsIV), a cycloartane triterpene saponin, is one of the major active ingredients of this plant Astragalus membranaceus (Huang Qi in Chinese). Several lines of evidence suggested that AsIV has remarkable cardioprotective effects via its anti-oxidative and anti-inflammatory activities $(15,16)$. AsIV also improved cardiac function through inhibiting cardiomyocyte hypertrophy and apoptosis $(13,15)$. In addition, the anti-fibrotic effect of AsIV was also seen in coxsackie virus-induced cardiomyopathy and in vitro cultured fibroblasts $(17,18)$. Since antioxidation represents as an important mechanism for the anti-proliferative effect of AsIV on cardiac fibroblasts (18), it is thus reckoned that CT-1 inhibition may also be involved in this pocess. Therefore, the aim of this study was to examine this possibility.

\section{Materials and methods}

Animal experiment. A total of 30 male heathy Sprague-Dawley rats, weighing 180-200 g were provided by the Animal Center, 
Jinzhou Medical University (Jinzhou, China). All rats were maintained in a temperature-controlled room $\left(25 \pm 0.2^{\circ} \mathrm{C}\right)$ with a 12/12 h light/dark cycle. These rats were fed with standard laboratory food and water. These rats were randomly divided into 3 groups $(n=10)$ : i) Control group, rats received the same volume of vehicle; ii) isoprenaline (ISO) group, rats received ISO injection $\left(10 \mathrm{mg} / \mathrm{kg}\right.$ day $^{-1}$ i.p.) for 4 weeks; and iii) ISO + AsIV group, rats received AsIV treatment $(80 \mathrm{mg} / \mathrm{kg}$ day $^{-1}$ i.g.) 2 weeks before 4-week ISO injection $(10 \mathrm{mg} / \mathrm{kg}$ day $^{-1}$ i.p.). ISO was dissolved in normal saline and AsIV in $1 \%$ sodium carboxymethyl cellulose solution. At the end of the treatment, left ventricular sections were prepared and stained with Sirius Red according to the instructions of the commercial kit (Beijing Leagene Biotech Co., Ltd., Beijing, China). All experimental procedures involving animals were conducted in accordance with the animal care guidelines of the National Institutes of Health (NIH) and Jinzhou Medical University. All efforts were made to minimize animal suffering and reduce the number of animals used.

CF culture. Primary cardiac fibroblasts (CFs) cultures were prepared from 1- to 3-day-old SD rats as our previously described method for culture of cardiomyocytes (12), except that pre-attached CFs but not cardiac myocytes were used for experiment. The cells were grown and maintained in Dulbecco's modified Eagle's medium (DMEM) (Corning Inc., Corning, NY, USA) containing 10\% fetal bovine serum (FBS; Hyclone; Thermo Fisher Scientific, Inc., Waltham, MA, USA) in $5 \% \mathrm{CO}_{2} / 95 \%$ air at $37^{\circ} \mathrm{C}$.

CF proliferation assay. CFs were planted and cultured in 96-well plates and challenged by ISO (Sigma-Aldrich; Merck KGaA (Darmstadt, Germany) following pretreatment with AsIV (>98\% purity; Nanjing Jingzhu Bio-technology Co., Ltd., Nanjing, China), $N$-acetylcysteine (NAC; $10 \mathrm{mM}$ ) and CT-1 siRNA transfection. DMEM (100 $\mu \mathrm{l})$ with $10 \mu \mathrm{l}$ CCK-8 (Dojindo Laboratories, Kumamoto, Japan) was included in each well for additional $4 \mathrm{~h}$ at $37^{\circ} \mathrm{C}$. The optical density was measured with an automated ELISA plate reader (Thermo Fisher Scientific, Inc., Waltham, MA, USA) at $450 \mathrm{~nm}$. Based on a previous study (4), a $24 \mathrm{~h}$-stimulation by ISO induced a significant elevation of CF proliferation.

Measurement of intracellular ROS. Intracellular ROS levels were detected by dihydroethidium (DHE; Molecular Probes, Eugene, OR, USA). CFs were first incubated in the dark with $10 \mu \mathrm{M}$ DHE for $1 \mathrm{~h}$ at $37^{\circ} \mathrm{C}$ in an incubator and then washed 3 times with phosphate-buffered saline (PBS). ROS level of the DHE loaded CFs was examined by an inverted fluorescence microscope (Leica, Wetzlar, Germany).

Knockdown of CT-1 by specific siRNA. Transfection was performed as previously reported (19), using siRNA sense sequence for CT-1, 5'-CCAAUUGCUGGAGGAAUAUtt-3', synthesized by GenePharma (Suzhou, China). To allow incorporation of CT-1 siRNA into CFs, CFs were incubated in serum-free DMEM for $24 \mathrm{~h}$ on the day before transfection. Transfection solution A containing $2.5 \mu \mathrm{l}(\sim 660 \mathrm{ng})$ siRNA and $40 \mu \mathrm{l}$ Opti-MEMI, and solution B containing $2 \mu \mathrm{l}$ Oliogofectamine and $5.5 \mu \mathrm{l}$ Opti-MEMI was firstly prepared and then mixed to form transfection solution $\mathrm{C}$ for use. The culturing medium was exchanged with $200 \mu 1$ DMEM without serum, with transfection solution $\mathrm{C}$ subsequently added and cultured at $37^{\circ} \mathrm{C}$ in a humidified atmosphere of $\mathrm{CO}_{2} /$ air (5:95\%) for $8 \mathrm{~h}$. Thereafter, $125 \mu \mathrm{l}$ DMEM supplemented with $30 \%$ serum was added to the cultures. Western blot analysis was performed to confirm the knockdown effect three days after transfection.

Quantitative real-time PCR. Total mRNA was extracted by Trizol agent (Invitrogen, Carlsbad, CA, USA). Reverse transcription was performed using a PrimeScript RT reagent kit (Takara Bio, Inc., Dalian, China) according to the manufacturer's instructions. RNA was then analyzed by Real-Time PCR using SYBR Premix Ex Taq ${ }^{\mathrm{TM}}$ (Takara Bio, Inc). The sequences of the primers used were as follows: CT-1 forward, 5'-GGAAGTCTGGAAGACCACCA-3' and reverse, 5'-TGC TGCACATATTCCTCCAG-3'; collagen I forward, 5'-TTC ACCTACAGCACGCTTGT-3' and reverse, 5'-TTGGGATGG AGGGAGTTTAC-3'; and GAPDH forward, 5'-TGGCCTCCA AGGAGTAAGAAAC-3' and reverse, 5'-GGCCTCTCTCTT GCTCTCAGTATC-3'. GAPDH gene was used as an internal control. PCR parameters were as follows: $95^{\circ} \mathrm{C}$ for $10 \mathrm{sec}$; 35 cycles of $95^{\circ} \mathrm{C}$ for $5 \mathrm{sec}$ and $60^{\circ} \mathrm{C}$ for $20 \mathrm{sec}$. PCR amplification was performed using the Mx3000P qPCR SYSTEM (Stratagene, La Jolla, CA, USA) and comparative Ct $(\Delta \Delta \mathrm{CT})$ method was used to determine the fold change in expression.

Western blotting. Total protein content was determined and equal amounts of protein were subjected to sodium dodecyl sulfate-polysacrylamide gel electrophoresis and blotted onto a polyvinylidene fluoride membrane. The membrane was blocked with $1 \%$ BSA for $1 \mathrm{~h}$ at room temperature and then incubated overnight at $4^{\circ} \mathrm{C}$ with the primary rabbit anti-rat polyclonal antibodies against collagen I (Col-I, 1:1,000, cat. no. ab34710; Abcam, Cambridge, MA, USA), $\beta$-actin (1:5,000, cat. no. 40552; Signalway Antibody, College Park, MD, USA) and mouse anti-rat monoclonal antibodies against CT-1 (1:1,000, cat. no. ab13975; Abcam). Following incubation with the polyclonal horseradish peroxidase-conjugated secondary goat anti-rabbit antibodies (1:2,500, cat. no. sc-2004) or goat anti-mouse antibodies (1:3,500, cat. no. sc-2005) (both from Santa Cruz Biotechnology, Inc., Dallas, TX, USA), the bands were detected by an enhanced chemiluminescence reagents (Thermo Fisher Scientific, Inc.). Quantityone software (version 4.6.9; Bio-Rad Laboratories, Inc., Hercules, CA, USA) was used to assess the visualized optical density for each band.

Statistical analysis. All data are shown as mean \pm standard error of mean (SEM) from at least three independent experiments. The data were subjected to one-way analysis of variance followed by Turkey's post hoc comparisons. The level of significance was set at $\mathrm{P}<0.05$.

\section{Results}

ISO induced CF proliferation was attenuated by increasing doses of AsIV. As shown in Fig. 1A, CF proliferation was significantly increased upon ISO stimulation for $24 \mathrm{~h}$. AsIV 
A

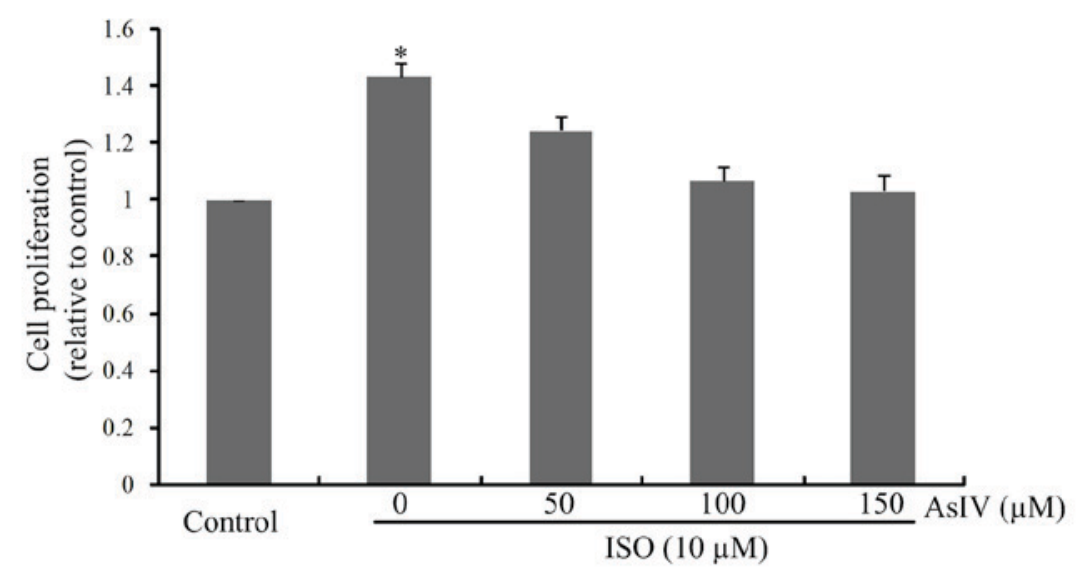

B

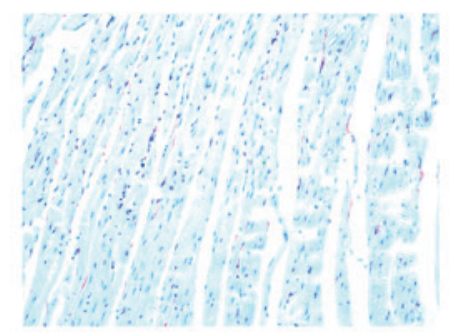

Control

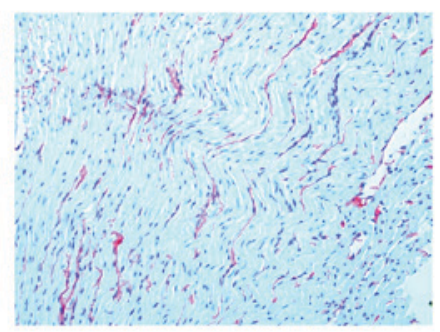

ISO

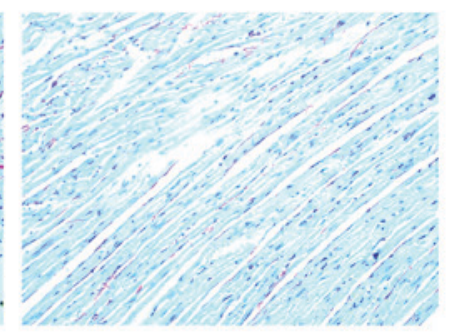

AsIV/ISO

Figure 1. Effect of astragaloside IV on isoproterenol-induced cardiac fibrosis in in vitro (A) and in vivo (B) model. Data are expressed as mean \pm standard error of mean (SEM) for five individual experiments. ${ }^{*} \mathrm{P}<0.05$ vs. control group.
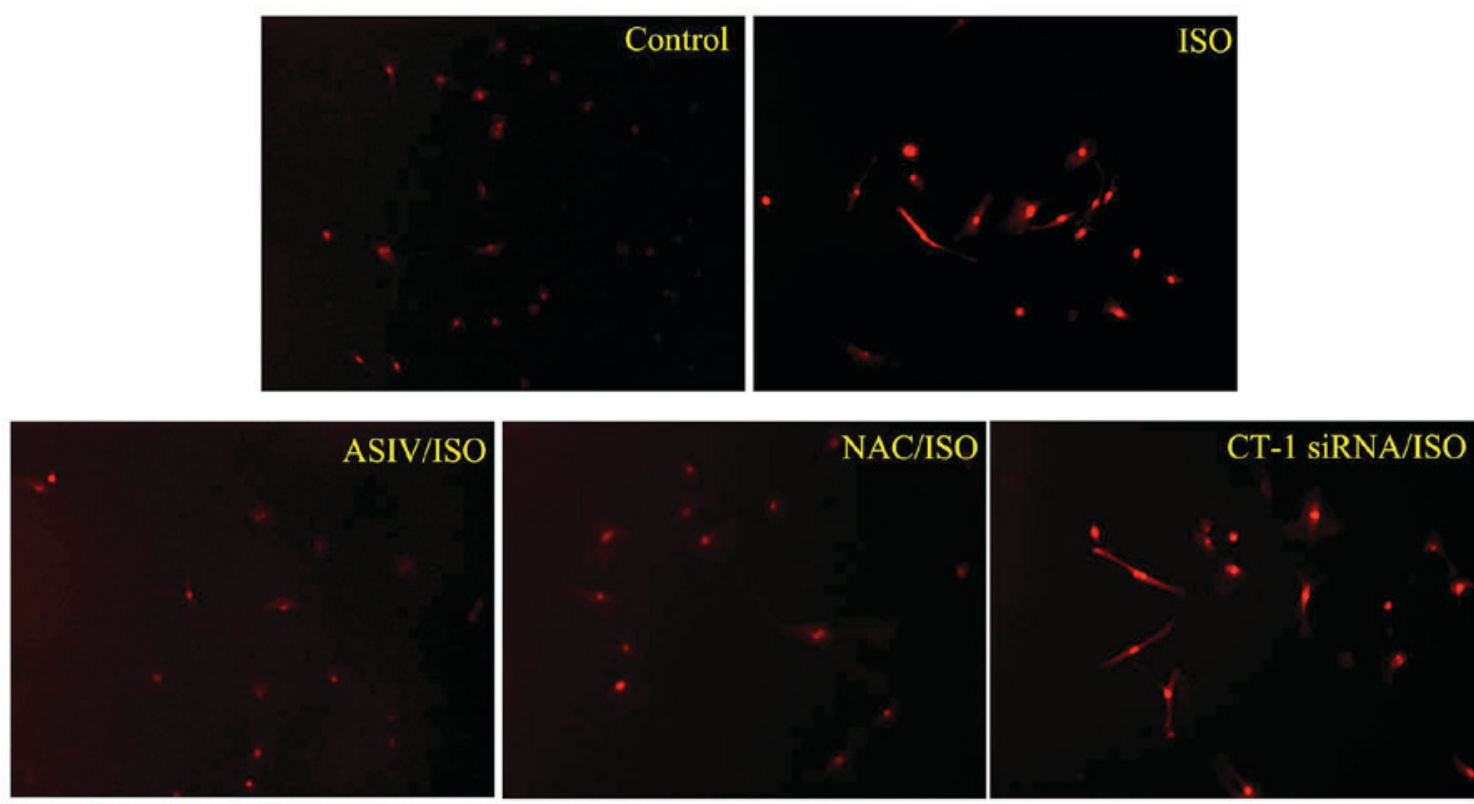

Figure 2. Effect of astragaloside IV (AsIV, $100 \mu \mathrm{M}$ ), $N$-acetylcysteine (NAC, $10 \mathrm{mM}$ ) and CT-1 siRNA on isoproterenol (ISO, $10 \mu \mathrm{M}$ )-induced reactive oxygen species (ROS) level in cardiofibroblasts (CFs).

pretreatment remarkably inhibited ISO triggered CF proliferation in a dose dependent manner. The anti-fibrotic effect of AsIV was further confirmed by Sirius Red stanning in vivo (Fig. 1B).

ISO induced ROS generation in CFs was decreased by AsIV, but not by CT-1 siRNA. As shown in Fig. 2, an elevated ROS production upon $30 \mathrm{~min}$ stimulation by ISO, which was effectively blunted by pretreatment with AsIV $(100 \mu \mathrm{M})$ or NAC $(10 \mathrm{mM})(20)$, the typical ROS scavenger. Great reduced expression of protein of CT-1 was confirmed by western blot analysis three days after transfection (data not shown). This transfection, however, failed to produce any effect on ISO-initiated ROS generation. 


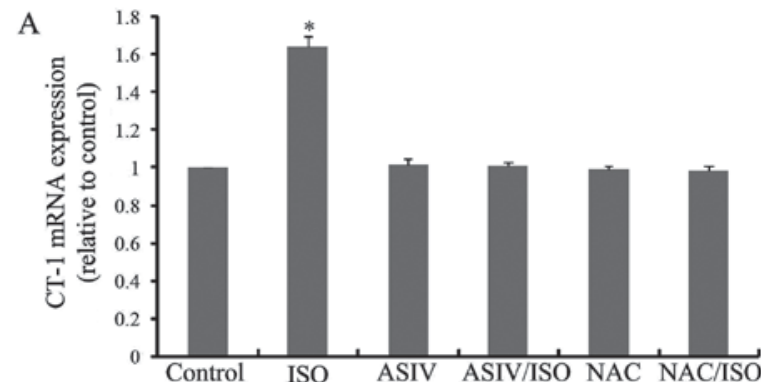

B

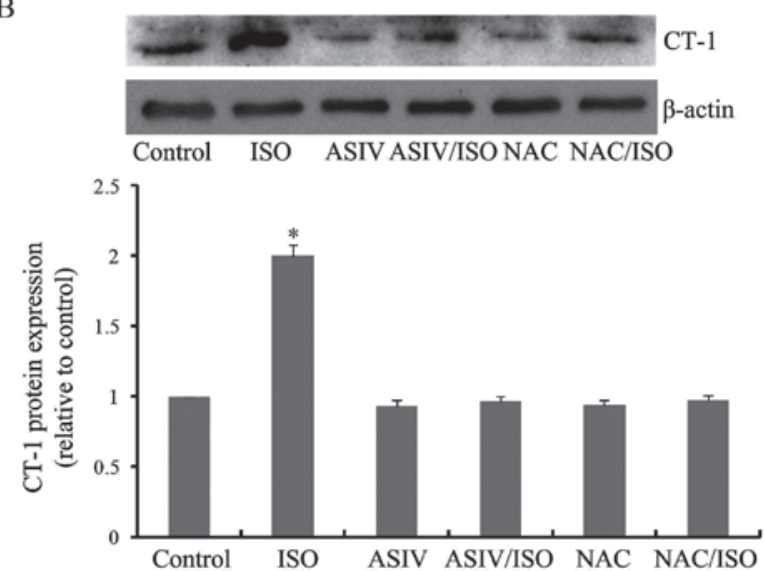

Figure 3. Effect of astragaloside IV (AsIV, $100 \mu \mathrm{M}$ ) and $N$-acetylcysteine (NAC, $10 \mathrm{mM}$ ) on isoproterenol (ISO, $10 \mu \mathrm{M}$ )-induced CT-1 overexpression at mRNA (A) and protein (B) levels. Data are expressed as mean \pm standard error of mean (SEM) for three individual experiments. "P<0.05 vs. other groups.

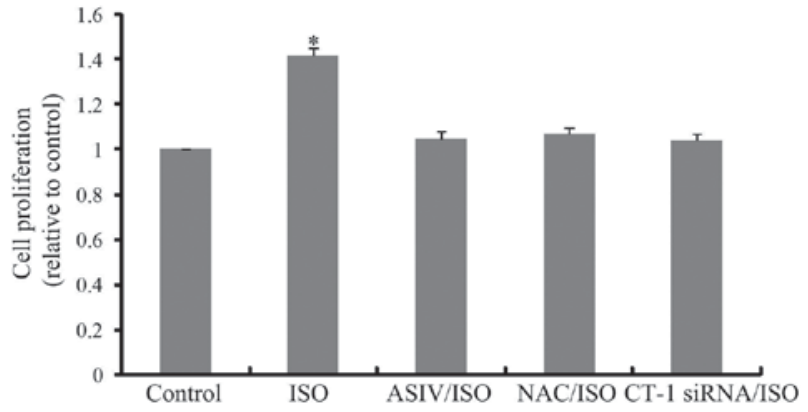

Figure 4. Effect of astragaloside IV (AsIV, $100 \mu \mathrm{M}$ ), $N$-acetylcysteine (NAC $10 \mathrm{mM}$ ) and CT-1 siRNA on isoproterenol (ISO, $10 \mu \mathrm{M}$ )-induced cardiac fibroblasts $(\mathrm{CFs})$ proliferation. Data are expressed as mean \pm standard error of mean (SEM) for five individual experiments. ${ }^{~} \mathrm{P}<0.05$ vs. other groups.

AsIV and NAC attenuated ISO induced overexpression of CT-1. As shown in Fig. 3A and B, ISO treatment for $24 \mathrm{~h}$ caused a significant elevation of CT-1 expression, both at mRNA and protein levels. These overexpressions of CT-1, however, were singnificantly inhibited by AsIV pretreatment. In addition, NAC produced similar inhibitory effects as AsIV on CT-1 overexpression.

CF proliferation and type I collagen synthesis induced by ISO was attenuated by AsIV, NAC and CT-1 siRNA pretreatment. As shown in Fig. 4, CF proliferation was significantly increased upon ISO stimulation for $24 \mathrm{~h}$. AsIV, NAC or CT-1 siRNA pretreatment remarkably inhibited ISO triggered CF

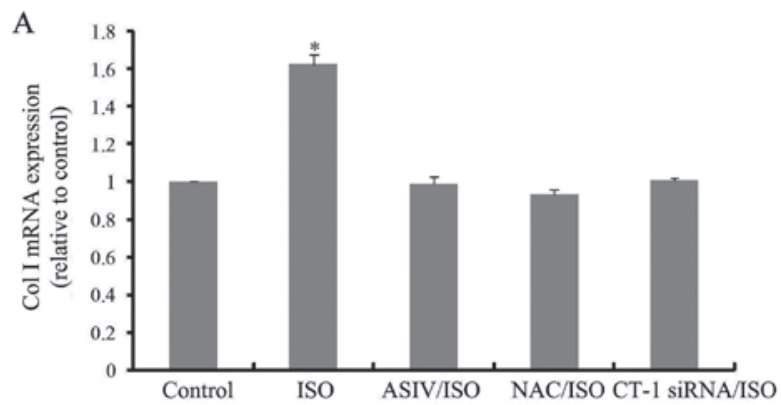

B
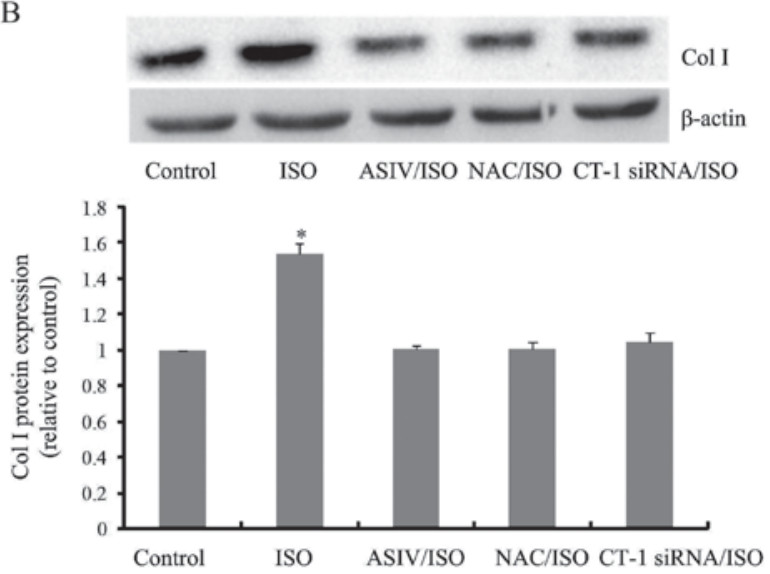

Figure 5. Effect of astragaloside IV (AsIV, $100 \mu \mathrm{M}$ ), $N$-acetylcysteine (NAC $10 \mathrm{mM}$ ) and CT-1 siRNA on isoproterenol (ISO, $10 \mu \mathrm{M}$ )-induced collagen expression at mRNA (A) and protein levels (B) in cardiac fibroblasts. Data are expressed as mean \pm standard error of mean (SEM) for three individual experiments. ${ }^{*} \mathrm{P}<0.05$ vs. other groups.

proliferation. Likewise, ISO-increased mRNA (Fig. 5A) and protein (Fig. 5B) expressions of collagen I, the most dominant component of extracellular matrix $(21,22)$, in cultured $\mathrm{CFs}$ were also blunted by application of AsIV, NAC or CT-1 siRNA.

\section{Discussion}

Cardiac fibrosis is a pivotal phenomenon and a hallmark in a variety of cardiovascular diseases (23). Enhanced CF proliferation and excess production and deposition of ECM represent the key characteristics of cardiac fibrosis. These pathological changes would eventually lead to myocardial stiffness, impaired diastolic function, severe arrhythmias, cardiac failure and even sudden cardiac death $(24,25)$. Therefore, early intervention in the fibrosis process would effectively slow down or prevent the procession of a variety of cardiovascular diseases.

Oxidative stress is a major pathogenesis mechanism for diverse cardiac disorders, including cardiac fibrosis, hypertrophy, apoptosis, inflammation, and resultant heart failure (26-28). It is evidenced that ROS could insult mitochondrial function and cause gene expression alteration both in cardiomyocytes and cardiofibroblasts $(29,30)$. Anti-oxidation can prevent the procession of cardiovascular events $(15,31,32)$. At present, the antioxidant property of AsIV has been demonstrated by many previous reports $(33,34)$. Intriguingly, we herein showed besides by NAC, ISO-invoked ROS overproduction was also blunted by application of 
AsIV, thereby suggestive of a possible protection effect of AsIV on ISO-induced damage of CFs via its anti-oxidation effect. Indeed, AsIV and NAC pretreatment also alleviated ISO-induce CF proliferation and collagen I synthesis. These data demonstrated an anti-oxidantion activity dependent anti-fibrotic effect of ASIV. It is unclear regarding how AsIV caused decreased production of ROS. In spite of that, our recent study showed that AsIV effectively suppressed the expression of mitochondrial NADPH oxidase 4 (mito-NOX4), and enhanced mitochondrial superoxide dismutase (mito-SOD) and mitochondrial catalase (mito-CAT) activity under ISO stimulation, both in intact heart tissue and in vitro cultured H9C2 cells (15). As such, it is reckoned that AsIV's antioxidant activity as shown here is probably related to its regulatory effect on mito-NOX4, mito-SOD, and mito-CAT, which needs further investigation.

CT-1 is a member belonging to IL-6 superfamily (35). By interacting with the heterodimer composed of glycoprotein 130 and leukemia inhibitory factor receptor- $\beta$, this cytokine exerts a series of cellular effects (36). Accumulating reports have delineated a key role for CT-1 in cardiomyocyte survival and hypertrophy, vascular smooth muscle cell (VSMC) proliferation, hypertrophy and extracellular matrix production. In addition, CT-1 is also involved in the pathogenesis of cardiac remodelling (37). Meanwhile, a direct stimulation of CT-1 on CF proliferation and collagen type I was also confirmed $(9,38)$. In the present study, a significant increase of CT-1 expression was seen in CFs upon ISO stimulation, which is in compatible with the statement that CFs are the predominant source of IL-6 in response to $\beta$-adrenergic receptor stimulation (39). CT-1 knockdown significantly reduced CF proliferation and collagen I production, suggesting an indispensible role of CT-1 in ISO-triggered cardiac fibrosis. As for the relationship between ROS and CT-1 pathways, our present study indicates that ISO-induced ROS production is needed for CT-1 overexpression, but not vice versa. A similar sequence as in the present study has been observed for effect of proxidant- and $\mathrm{CoCl}_{2}$-mediated upregulation of CT-1 in mouse embryonic stem cells (11). Both ROS production and CT-1 overexpression triggered by ISO were remarkably suppressed by AsIV, thereby revealing an ROS-CT-1-targeted anti-fibrotic effect of this natural occurring substance. Since ROS operated upstream of CT-1 overexpression upon ISO stimulation, and AsIV would effectively blunted ROS production, it is argued that AsIV reduce CT-1 overexpression via, at least in part, its antioxidant activity. But we are still unable to exclude the possibility that AsIV may also have direct inhibitory effect on CT-1 overexpression induced by ISO.

A. membranaceus has been widely used in traditional Chinese medicine for the treatment of cardiovascular diseases and AsIV represents one of the major active ingredients thereof. Previous researches mainly paid more attention to the cardiomyocytes, such as their hypertrophy or apoptosis, than cardiofibroblasts $(\mathrm{CFs})(15,40)$. In contrast, our previous study has confirmed the beneficial effect of AsIV on in vitro cultured CFs, including that of anti-oxidation and anti-fibrosis (18). Our present study herein further revealed that the anti-fibrotic effect of AsIV may be related to ROS-mediated CT-1 overexpression. Although it is unclear regarding how AsIV exerted its anti-fibrotic effect, $\beta$-receptor inhibition seems not involved in this process, as AsIV is also able to prevent cardiac fibrosis induced by stimuli irrelevant to $\beta$-receptor activation, such as coxsackievirus (17). This finding, together with other findings, would provide us comprehensive understanding of the cardioprotective effect of this natural occurring substance. And also, our present finding suggests that AsIV may serve as a useful therapeutic treatment in patients with fibrosis- and remodelling-related cardiovascular disorders, such as chronic heart failure, diabetic cardiomyopathy, and iron overload cardiomyopathy (41-43).

\section{Acknowledgements}

This study was carried out with the support of National Natural Science Foundation of China (grant no. 81673632).

\section{References}

1. Yao Y, Shen D, Chen R, Ying C, Wang C, Guo J and Zhang G: Galectin-3 predicts left ventricular remodeling of hypertension. J Clin Hypertens (Greenwich) 18: 506-511, 2016.

2. Zhou Y, Fang H, Lin S, Shen S, Tao L, Xiao J and Li X: Qiliqiangxin protects against cardiac ischemia-reperfusion injury via activation of the mTOR pathway. Cell Physiol Biochem 37: 454-464, 2015.

3. Decker JA: Arrhythmias in paediatric valvar disease. Cardiol Young 24: 1064-1070, 2014.

4. Lu H, Tian A, Wu J, Yang C, Xing R, Jia P, Yang L, Zhang Y, Zheng $X$ and Li $Z$ : Danshensu inhibits $\beta$-adrenergic receptors-mediated cardiac fibrosis by ROS/p38 MAPK axis. Biol Pharm Bull 37: 961-967, 2014.

5. Löfsjögård J, Persson H, Díez J, López B, González A, Edner M, Mejhert $M$ and Kahan T: Atrial fibrillation and biomarkers of myocardial fibrosis in heart failure. Scand Cardiovasc J 48 : 299-303, 2014.

6. Sirish P, Li N, Liu JY, Lee KS, Hwang SH, Qiu H, Zhao C, Ma SM, López JE, Hammock BD and Chiamvimonvat N: Unique mechanistic insights into the beneficial effects of soluble epoxide hydrolase inhibitors in the prevention of cardiac fibrosis. Proc Natl Acad Sci USA 110: 5618-5623, 2013.

7. Yang L, Zou XJ, Gao X, Chen H, Luo JL, Wang ZH, Liang QS and Yang GT: Sodium tanshinone IIA sulfonate attenuates angiotensin II-induced collagen type I expression in cardiac fibroblasts in vitro. Exp Mol Med 41: 508-516, 2009.

8. Freed DH, Chilton L, Li Y, Dangerfield AL, Raizman JE, Rattan SG, Visen N, Hryshko LV and Dixon IM: Role of myosin light chain kinase in cardiotrophin-1-induced cardiac myofibroblast cell migration. Am J Physiol Heart Circ Physiol 301: H514-H522, 2011.

9. Freed DH, Borowiec AM, Angelovska T and Dixon IM: Induction of protein synthesis in cardiac fibroblasts by cardiotrophin-1: Integration of multiple signaling pathways. Cardiovasc Res 60: 365-375, 2003.

10. López B, González A, Querejeta R, Larman M, Rábago G and Díez J: Association of cardiotrophin-1 with myocardial fibrosis in hypertensive patients with heart failure. Hypertension 63: 483-489, 2014.

11. Ateghang B, Wartenberg M, Gassmann M and Sauer H: Regulation of cardiotrophin-1 expression in mouse embryonic stem cells by HIF-1alpha and intracellular reactive oxygen species. J Cell Sci 119: 1043-1052, 2006.

12. Dai H, Jia G, Liu X, Liu Z and Wang H: Astragalus polysaccharide inhibits isoprenaline-induced cardiac hypertrophy via suppressing $\mathrm{Ca}^{2+}$-mediated calcineurin/NFATc3 and CaMKII signaling cascades. Environ Toxicol Pharmacol 38: 263-271, 2014.

13. Yang J, Wang HX, Zhang YJ, Yang YH, Lu ML, Zhang J, Li ST, Zhang SP and Li G: Astragaloside IV attenuates inflammatory cytokines by inhibiting TLR4/NF-кB signaling pathway in isoproterenol-induced myocardial hypertrophy. J Ethnopharmacol: Oct 25, 2013 (Epub ahead of print).

14. Jiang X, Cao X, Huang Y, Chen J, Yao X, Zhao M, Liu Y, Meng J, Li P, Li Z, et al: Effects of treatment with Astragalus membranaceus on function of rat leydig cells. BMC Complement Altern Med 15: 261, 2015. 
15. Mei M, Tang F, Lu M, He X, Wang H, Hou X, Hu J, Xu C and Han R: Astragaloside IV attenuates apoptosis of hypertrophic cardiomyocyte through inhibiting oxidative stress and calpain-1 activation. Environ Toxicol Pharmacol 40: 764-773, 2015.

16. Zhao P, Wang Y, Zeng S, Lu J, Jiang TM and Li YM: Protective effect of astragaloside IV on lipopolysaccharide-induced cardiac dysfunction via downregulation of inflammatory signaling in mice. Immunopharmacol Immunotoxicol 37: 428-433, 2015.

17. Chen P, Xie Y, Shen E, Li GG, Yu Y, Zhang CB, Yang Y, Zou Y, Ge J, Chen R and Chen H: Astragaloside IV attenuates myocardial fibrosis by inhibiting TGF- $\beta 1$ signaling in coxsackievirus B3-induced cardiomyopathy. Eur J Pharmacol 658: 168-174, 2011.

18. Dai H, Jia G, Lu M, Liang C, Wang Y and Wang H: Astragaloside IV inhibits isoprenaline-induced cardiac fibrosis by targeting the reactive oxygen species/mitogen-activated protein kinase signaling axis. Mol Med Rep 15: 1765-1770, 2017.

19. Dai H, Song D, Xu J, Li B, Hertz L and Peng L: Ammonia-induced $\mathrm{Na}, \mathrm{K}-\mathrm{ATP}$ ase/ouabain-mediated EGF receptor transactivation, MAPK/ERK and PI3K/AKT signaling and ROS formation cause astrocyte swelling. Neurochem Int 63: 610-625, 2013.

20. Yu M, Zheng Y, Sun HX and Yu DJ: Inhibitory effects of enalaprilat on rat cardiac fibroblast proliferation via ROS/P38MAPK/TGF- $\beta 1$ signaling pathway. Molecules 17: 2738-2751, 2012

21. Porter KE and Turner NA: Cardiac fibroblasts: At the heart of myocardial remodeling. Pharmacol Ther 123: 255-278, 2009.

22. Ye Y, Lv X, Wang MH, Zhu J, Chen SQ, Jiang CY and Fu GS: Alendronate prevents angiotensin II-induced collagen I production through geranylgeranylation-dependent RhoA/Rho kinase activation in cardiac fibroblasts. J Pharmacol Sci 129: 205-209, 2015.

23. Yu LM and Xu Y: Epigenetic regulation in cardiac fibrosis. World J Cardiol 7: 784-791, 2015.

24. van Putten S, Shafieyan Y and Hinz B: Mechanical control of cardiac myofibroblasts. J Mol Cell Cardiol 93: 133-142, 2016

25. Chimura M, Kiuchi K, Okajima K, Shimane A, Sawada T, Onishi T, Yamada S, Taniguchi Y, Yasaka Y and Kawai H: Distribution of ventricular fibrosis associated with life threatening ventricular tachyarrhythmias in patients with nonishcemic dilated cardiomyopathy. J Cardiovasc Electrophysiol: Jul 29, 2015 (Epub ahead of print).

26. Wu H, Li GN, Xie J, Li R, Chen QH, Chen JZ, Wei ZH, Kang LN and $\mathrm{Xu} \mathrm{B}$ : Resveratrol ameliorates myocardial fibrosis by inhibiting ROS/ERK/TGF- $\beta /$ periostin pathway in STZ-induced diabetic mice. BMC Cardiovasc Disord 16: 5, 2016.

27. Ghule AE, Kandhare AD, Jadhav SS, Zanwar AA and Bodhankar SL: Omega-3-fatty acid adds to the protective effect of flax lignan concentrate in pressure overload-induced myocardial hypertrophy in rats via modulation of oxidative stress and apoptosis. Int Immunopharmacol 28: 751-763, 2015.

28. An LP, An SK, Wei XH, Fu SY and Wu HA: Atorvastatin improves cardiac function of rats with chronic cardiac failure via inhibiting Rac1/P47 ${ }^{\text {phox }} / \mathrm{P} 67^{\text {phox }}$-mediated ROS release. Eur Rev Med Pharmacol Sci 19: 3940-3946, 2015

29. Bartz RR, Suliman HB and Piantadosi CA: Redox mechanisms of cardiomyocyte mitochondrial protection. Front Physiol 6: 291, 2015.

30. Schunke KJ, Coyle L, Merrill GF and Denhardt DT: Acetaminophen attenuates doxorubicin-induced cardiac fibrosis via osteopontin and GATA4 regulation: Reduction of oxidant levels. J Cell Physiol 228: 2006-2014, 2013.
31. Chen O, Ye Z, Cao Z, Manaenko A, Ning K, Zhai X, Zhang R, Zhang T, Chen X, Liu W and Sun X: Methane attenuates myocardial ischemia injury in rats through anti-oxidative, anti-apoptotic and anti-inflammatory actions. Free Radic Biol Med 90: 1-11, 2016.

32. Mathur P, Ding Z, Saldeen T and Mehta JL: Tocopherols in the prevention and treatment of atherosclerosis and related cardiovascular disease. Clin Cardiol 38: 570-576, 2015.

33. Gu DM, Lu PH, Zhang K, Wang X, Sun M, Chen GQ and Wang Q: EGFR mediates astragaloside IV-induced Nrf2 activation to protect cortical neurons against in vitro ischemia/reperfusion damages. Biochem Biophys Res Commun 457: 391-397, 2015.

34. Hu JY, Han J, Chu ZG, Song HP, Zhang DX, Zhang Q and Huang YS: Astragaloside IV attenuates hypoxia-induced cardiomyocyte damage in rats by upregulating superoxide dismutase-1 levels. Clin Exp Pharmacol Physiol 36: 351-357, 2009.

35. Zheng ZZ, Tian Fu X, Liang J and Bing Guo Z: CT-1 induces angiogenesis by regulating the ADMA/DDAH pathway. Biomed Pap Med Fac Univ Palacky Olomouc Czech Repub 159: 540-546, 2015.

36. Pennica D, King KL, Shaw KJ, Luis E, Rullamas J, Luoh SM, Darbonne WC, Knutzon DS, Yen R, Chien KR, et al: Expression cloning of cardiotrophin 1, a cytokine that induces cardiac myocyte hypertrophy. Proc Natl Acad Sci USA 92: 1142-1146, 1995.

37. López-Andrés N, Martin-Fernandez B, Rossignol P, Zannad F, Lahera V, Fortuno MA, Cachofeiro V and Díez J: A role for cardiotrophin-1 in myocardial remodeling induced by aldosterone. Am J Physiol Heart Circ Physiol 301: H2372-H2382, 2011.

38. Tsuruda T, Jougasaki M, Boerrigter G, Huntley BK, Chen HH, D'Assoro AB, Lee SC, Larsen AM, Cataliotti A and Burnett JC Jr: Cardiotrophin-1 stimulation of cardiac fibroblast growth: Roles for glycoprotein 130/leukemia inhibitory factor receptor and the endothelin type A receptor. Circ Res 90: 128-134, 2002.

39. Yin F, Li P, Zheng M, Chen L, Xu Q, Chen K, Wang YY, Zhang YY and Han C: Interleukin-6 family of cytokines mediates isoproterenol-induced delayed STAT3 activation in mouse heart. J Biol Chem 278: 21070-21075, 2003.

40. Zhang S, Tang F, Yang Y, Lu M, Luan A, Zhang J, Yang J and Wang H: Astragaloside IV protects against isoproterenol-induced cardiac hypertrophy by regulating NF- $\kappa \mathrm{B} / \mathrm{PGC}-1 \alpha$ signaling mediated energy biosynthesis. PLoS One 10: e0118759, 2015.

41. Giam B, Chu PY, Kuruppu S, Smith AI, Horlock D, Kiriazis H, Du XJ, Kaye DM and Rajapakse NW: $N$-acetylcysteine attenuates the development of cardiac fibrosis and remodeling in a mouse model of heart failure. Physiol Rep 4: e12757, 2016.

42. Faramoushi M, Amir Sasan R, Sari Sarraf V and Karimi P: Cardiac fibrosis and down regulation of GLUT4 in experimental diabetic cardiomyopathy are ameliorated by chronic exposures to intermittent altitude. J Cardiovasc Thorac Res 8: 26-33, 2016.

43. Zhang Y, Wang H, Cui L, Zhang Y, Liu Y, Chu X, Liu Z, Zhang J and Chu L: Continuing treatment with Salvia miltiorrhiza injection attenuates myocardial fibrosis in chronic iron-overloaded mice. PLoS One 10: e0124061, 2015. 